JOURNAL OF SYNCHROTRON RADIATION

ISSN 1600-5775

Received 7 August 2019

Accepted 17 February 2020

Edited by A. Bergamaschi, Paul Scherrer Institut, Switzerland

₹ Now at: Division of Biology and Chemistry, Laboratory for Biomolecular Research, Paul Scherrer Institute, 5232 Villigen, Switzerland.

Keywords: diffraction patterns; LiF detectors; color centers.



C 2020 International Union of Crystallography

\section{Soft X-ray diffraction patterns measured by a LiF detector with sub-micrometre resolution and an ultimate dynamic range}

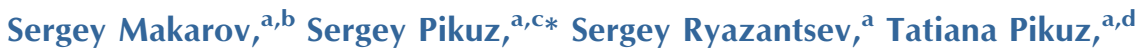 \\ Alexey Buzmakov, ${ }^{\text {e }}$ Max Rose, ${ }^{\mathfrak{f}}$ Sergey Lazarev, ${ }^{\mathrm{f}, \mathrm{g}}$ Tobias Senkbeil, ${ }^{\text {, }}$ \\ Andreas von Gundlach, ${ }^{\text {h }}$ Susan Stuhr, ${ }^{\text {h }}$ Christoph Rumancev, ${ }^{\text {h }}$ \\ Dmitry Dzhigaev, ${ }^{f, i}$ Petr Skopintsev, ${ }^{f} \ddagger$ Ivan Zaluzhnyy, ${ }^{f, c}$ \\ Jens Viefhaus, ${ }^{f}$ Axel Rosenhahn, ${ }^{\text {h }}$ Ryosuke Kodama ${ }^{j}$ and Ivan A. Vartanyants ${ }^{c, f}$
}

\begin{abstract}
aJoint Institute for High Temperatures, Russian Academy of Sciences, Izhorskaya Street 13 Bd 2, Moscow 125412, Russian Federation, 'b ${ }^{\mathbf{S}}$ kobeltsyn Institute of Nuclear Physics, Lomonosov Moscow State University, Leninskie gory, GSP-1, Moscow 119991, Russian Federation, ' Moscow Engineering Physics Institute (MEPhl), Kashirskoe shosse 31, Moscow 115409, Russian Federation, ${ }^{\mathbf{d}}$ Institute for Open and Transdisciplinary Research Initiatives, Osaka University, 2-6 Yamadaoka, Suita, Osaka 565-0871, Japan, ${ }^{\mathbf{e}}$ Russian Academy of Sciences, Federal Research Centre Crystallography and Photonics, Leninskii pr-t 59, Moscow 119333, Russian Federation, 'Deutsches ElektronenSynchrotron (DESY), Notkestrasse 85, Hamburg 22607, Germany, ${ }^{\mathbf{g}}$ National Research Tomsk Polytechnic University (TPU), 30 Lenin Avenue, Tomsk 634050, Russian Federation, hAnalytical Chemistry - Biointerfaces, Ruhr University Bochum, Universitatsstrasse 150, Bochum 44780, Germany, 'Division of Synchrotron Radiation Research, Department of Physics, Lund University, Box 118, SE-221 00 Lund, Sweden, and I'Institute of Laser Engineering, Osaka University, 2-6 Yamadaoka, Suita, Osaka 565-0871, Japan. *Correspondence e-mail: spikuz@gmail.com
\end{abstract}

The unique diagnostic possibilities of X-ray diffraction, small X-ray scattering and phase-contrast imaging techniques applied with high-intensity coherent $\mathrm{X}$-ray synchrotron and X-ray free-electron laser radiation can only be fully realized if a sufficient dynamic range and/or spatial resolution of the detector is available. In this work, it is demonstrated that the use of lithium fluoride (LiF) as a photoluminescence (PL) imaging detector allows measuring of an X-ray diffraction image with a dynamic range of $\sim 10^{7}$ within the sub-micrometre spatial resolution. At the PETRA III facility, the diffraction pattern created behind a circular aperture with a diameter of $5 \mu \mathrm{m}$ irradiated by a beam with a photon energy of $500 \mathrm{eV}$ was recorded on a $\mathrm{LiF}$ crystal. In the diffraction pattern, the accumulated dose was varied from $1.7 \times 10^{5} \mathrm{~J} \mathrm{~cm}^{-3}$ in the central maximum to $2 \times 10^{-2} \mathrm{~J} \mathrm{~cm}^{-3}$ in the 16 th maximum of diffraction fringes. The period of the last fringe was measured with $0.8 \mu \mathrm{m}$ width. The PL response of the $\mathrm{LiF}$ crystal being used as a detector on the irradiation dose of $500 \mathrm{eV}$ photons was evaluated. For the particular model of laser-scanning confocal microscope Carl Zeiss LSM700, used for the readout of the PL signal, the calibration dependencies on the intensity of photopumping (excitation) radiation $(\lambda=488 \mathrm{~nm})$ and the gain have been obtained.

\section{Introduction}

Various diagnostic techniques based on X-ray radiation such as coherent X-ray diffractive imaging, ptychographic coherent diffractive imaging and small-angle X-ray scattering are broadly used these days for studying matter at the nanometre scale. The advent of next-generation synchrotron sources as well as X-ray free-electron lasers (XFELs) delivering highly coherent and ultrabright radiation opens unique opportunities to study ultrafast processes in matter including various high energy-density physics (HEDP) phenomena. In turn, it imposes additional requirements on X-ray detection systems to be applied in such studies. In particular, the detector should be sustainable to ultraintense X-ray fluxes and strong electromagnetic pulses (EMPs). 
One of the most important requirements for a detector in such types of experiment is the simultaneous support of submicrometre spatial resolution, a wide dynamic range and a large field of view. The use of conventional detectors often cannot satisfy this requirement when recording images at intense X-ray fields.

While commonly used CCD cameras may provide a high dynamic range (up to $1 \times 10^{4}$ ) and tremendous sensitivity where almost every single photon can be detected in a realtime acquisition mode, these detectors are quite fragile to overexposure and unsustainable to EMPs, and their use in HEDP experiments is often restricted.

The issue can be mitigated with the application of analog detectors such as image plates (IPs), scintillator screens or $\mathrm{X}$-ray films, though they are less convenient for real-time data acquisition. However, they provide a limited spatial resolution (not better than $25 \mu \mathrm{m}$ for IPs and 4 to $5 \mu \mathrm{m}$ for X-ray films) and their dynamic range does not exceed $10^{5}$ for a single exposure.

A relatively new and promising X-ray detector is lithium fluoride ( $\mathrm{LiF}$ ) in the form of bulk crystal and thin film, which has demonstrated its performance as a 2D and 3D imaging tool in a wide range of applications including X-ray microradiographs of small biological objects in the water-window spectral range (energy range between the absorption edges of carbon and oxygen at $284 \mathrm{eV}$ and $532 \mathrm{eV}$ ) (Baldacchini et al., 2003); visualization and characterization of focused X-ray beams (Faenov et al., 2009; Pikuz et al., 2015; Bonfigli et al., 2016); coherent X-ray beam metrology by diffraction method (Ruiz-Lopez et al., 2017); X-ray radiography with a large aspect ratio field-of-view/spatial resolution; and, in particular, in the interests of HEDP (Faenov et al., 2018), phase-contrast imaging and quality control of nano-thickness foils (Faenov et al., 2010; Pikuz et al., 2018) and space-resolved dosimetry (Kurobori \& Matoba, 2014).

The principle of the operation with the $\mathrm{LiF}$ detector is shown in Fig. 1. When a photon with energy exceeding $14 \mathrm{eV}$ propagates in the medium, the absorbed energy moves electrons from the valence band to the conduction band of the crystal. Free electrons occupy anion vacancies in crystal cells and create so-called F color centers (CCs). During the relaxation process, primary $\mathrm{F}$ centers aggregate to more complex CC structures containing several excited centers. Among various aggregates of $\mathrm{CCs}$, only $\mathrm{F}_{2}$ and $\mathrm{F}_{3}^{+}$have practical importance for imaging applications. They are metastable and have almost overlapping broad absorption bands at $\sim 450 \mathrm{~nm}$ [see Fig. 1(c)]. This means that they can be simultaneously excited by a single pumping wavelength. Moreover, they mainly contribute to visible luminescence in two well-separated broad emission bands in

Figure 1 the green $\left(\mathrm{F}_{3}^{+}\right)$and in the red $\left(\mathrm{F}_{2}\right)$ spectral ranges [see Fig. $1(c)$ ]. It is essential that CCs are stable at room temperature, so the hidden image formed within the $\mathrm{LiF}$ volume is kept for many years almost without decay. In comparison, the lifetime of stored CCs in IPs is of a few hours and the corresponding readout process must be carried out immediately after the exposure. Note that these centers can also be generated by protons (Piccinini et al., 2017; Montereali et al., 2018), neutrons (Faenov et al., 2015), electrons (Almaviva et al., 2007), and $\gamma$ and X-ray photons (Kurobori et al., 2014; Voitovich et al., 2013).

To finalize the X-ray detection procedure (Fig. 1), the distribution of CCs can be conveniently observed by a fluorescent optical microscope. For recounting of the microscope photoluminescence (PL) signal into the accumulated dose in the crystal, one should know the microscope apparatus functions [see the red rectangle in Fig. $1(d)$ ] as well as the dependence of the CCs number on the absorbed dose [the green rectangle in Fig. 1(d)]. In this article, we follow from the microscope signal to the real accumulated dose of the $\mathrm{LiF}$ crystal.

The LiF detector does not require any electronic circuits and therefore is not sensitive to electromagnetic noise, which can be an issue for the use of CCD cameras. The disadvantage of the LiF detector is in its inability to display the image in real time, at least with existing microscope readout systems, until a special fiber-connected fluorescent microscope head can be built. The total time to obtain an image using this detector is comparable with the readout time for the IPs. A typical scan of the image $(512 \times 512$ pixels $)$ takes tens of seconds, depending on the scanning conditions. However, when the experimental setup employs a vacuum vessel, the most time-consuming

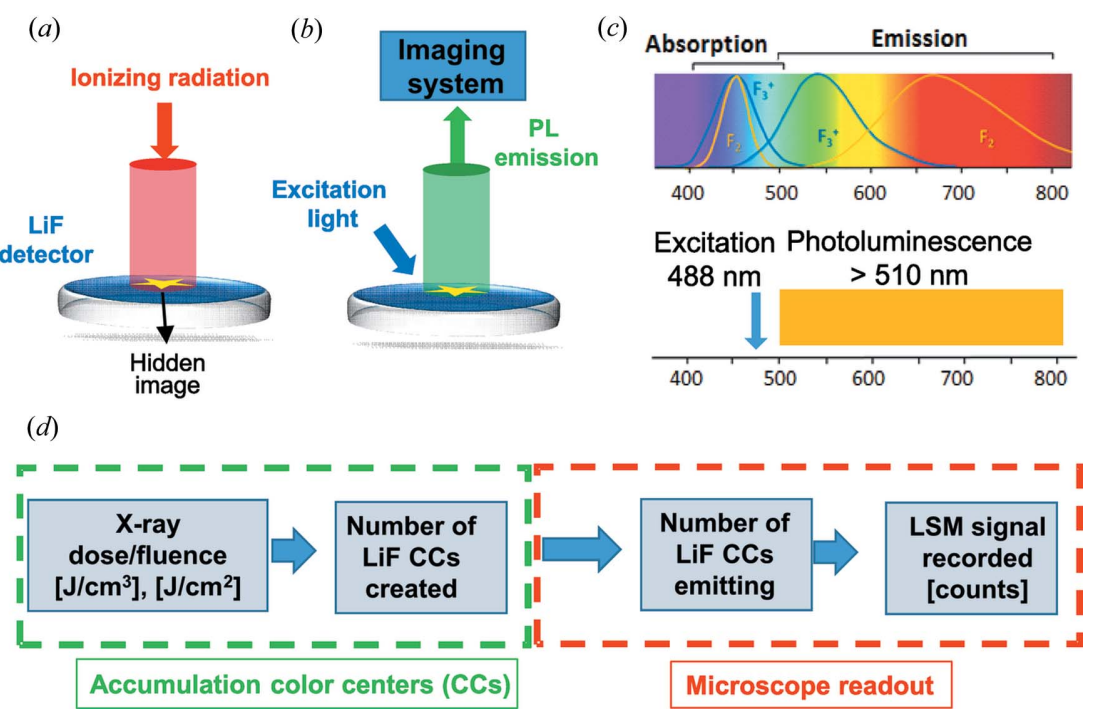

Principle of the operation with a $\mathrm{LiF}$ detector. (a) Irradiation of a $\mathrm{LiF}$ crystal by ionizing radiation with an energy above $14 \mathrm{eV}$. (b) Visualization of a CCs distribution into a LiF volume using an optical system with excitation light (e.g. an optical microscope in fluorescent mode) (c) Absorption and emission bands for $\mathrm{F}_{2}$ and $\mathrm{F}_{3}^{+}$CCs in the LiF crystal. (d) Steps of the readout procedure via confocal fluorescence microscope for determining the dose accumulated in the $\mathrm{LiF}$ detector. 
readout stage is the venting and opening of the vacuum chamber which may cost $\sim 1 \mathrm{~h}$ of operation.

Characterization of the metrological properties of the $\mathrm{LiF}$ crystals such as sensitivity, dynamic range and the spatial resolution is an important prerequisite when using it as an $\mathrm{X}$-ray detector. There have been several studies of such functions for hard X-rays (Pikuz et al., 2015; Grum-Grzhimailo et al., 2017; Bonfigli et al., 2019), while the data in a soft spectral range were previously measured only by using a laserplasma source (Baldacchini et al., 2005), and there are no data on the dependence of LiF PL response to narrow-bandwidth quasi-monochromatic radiation. The main aim of the present work is in the precise characterization of the response function, sensitivity threshold and dynamic range of the $\mathrm{LiF}$ detector in the vicinity of the water-window band. The performance of the $\mathrm{LiF}$ detector is demonstrated by the measurement of soft-X-ray diffraction patterns with submicrometre resolution and the ultimate dynamic range.

\section{Experimental method}

The experiment was performed at the soft X-ray beamline P04 of the third-generation synchrotron source PETRA III at DESY, Hamburg, Germany (Viefhaus et al., 2013). An APPLE-II type helical undulator of length $5 \mathrm{~m}$ with 72 magnetic periods was tuned to deliver photons at an energy of $500 \mathrm{eV}$ (Rose et al., 2015). The X-ray beam was focused by the two-mirror system in vertical and horizontal directions. The measurements were carried out in the holographic Roentgen scattering (HORST) vacuum chamber (Rose et al., 2018).

As mentioned above, knowledge of both the response function and the dynamic range of the LiF detector is important for a successful application. In the first phase of the experiment, we investigated the response function of $\mathrm{LiF}$ crystal to soft X-ray radiation. For this, the diffraction pattern behind a slit was captured by a LiF crystal and then it was compared with data from a calibrated photodiode. A sketch of the experimental setup is shown in Fig. 2(a). Both the diode and the LiF crystal were mounted on a single motorized scanning stage with nanometre precision. A simple translation motion was therefore sufficient in order to choose to send the synchrotron radiation to either the diode or the LiF crystal. First, the integral photon flux incident on the LiF detector position was measured by a diode as $\sim 10^{9}$ photons per second. The diode was calibrated with a monochromatic source of known intensity prior to the experiment. The area of the beam was limited to a slit gap with a size of $100 \mu \mathrm{m}$. The diffraction pattern was recorded at a distance of $1 \mathrm{~m}$ from the slit. The whole image of the pattern was measured by the $\mathrm{LiF}$ detector in a field of view of $2 \mathrm{~mm}$ and with an exposure time of $180 \mathrm{~s}$. Additionally, the 2D intensity profile was measured by scanning the photodiode through the center of the diffraction pattern in horizontal and vertical directions. The field of view of the photodiode was limited by a pinhole of size $1.5 \mu \mathrm{m}$.

In the second step of the experiment, a series of measurements using the slit and the pinhole were conducted to identify the dynamic range of the crystal and to demonstrate the (a)

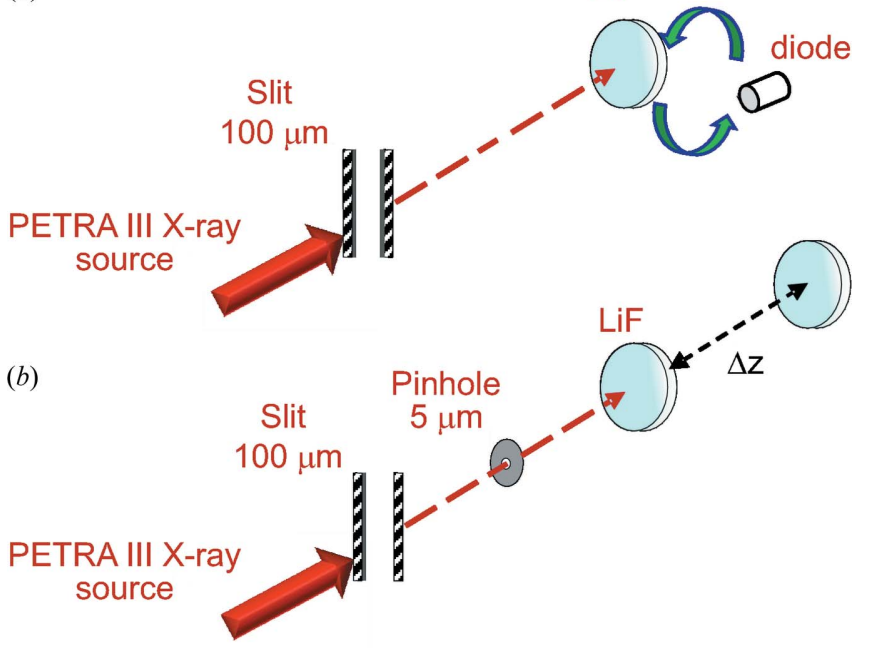

Figure 2

Two cases of the experimental setup. (a) The X-ray beam propagated through the slit and its profile was measured alternatively by the $\mathrm{LiF}$ crystal detector or the pre-calibrated photodiode. (b) Scanning of the intensity distribution of the X-ray beam propagated through both the slit and the pinhole in different planes along the $Z$ axis using the LiF crystal detector.

imaging capability of LiF [see Fig. 2(b)]. The intensity distribution of the synchrotron beam was measured at sequences of planes. For that purpose, the LiF crystal was moved along the $Z$ axis in the range 1 to $12 \mathrm{~mm}$ (an axis along the beam propagation direction).

After the experiments, the LiF crystals were analyzed by a confocal laser-scanning microscope (model Carl Zeiss LSM700). The PL signal from the irradiated crystal was recorded by a 16-bit photomultiplier (PMT). The PL intensity was controlled by the PMT voltage (parameter gain) and the level of excitation radiation (the LSM laser power). The calibration parameters were obtained as a function of the intensity of photopumping (excitation) radiation $(\lambda=488 \mathrm{~nm})$ and the gain for the particular model of laser-scanning confocal microscope. A detailed description of the calibration procedure is presented in the Appendix $A$.

It is known that only $\mathrm{F}_{2}$ and $\mathrm{F}_{3}^{+} \mathrm{CCs}$ are metastable and emit in the visible range [see Fig. 1(b)], i.e. giving the contribution to the LSM signal. There is no general way to evaluate the dose quantitatively as a function of emitting CCs number. However, we were able to excite mainly $\mathrm{F}_{2}$ type of $\mathrm{CCs}$ via the $488 \mathrm{~nm}$ pumping laser, where the $F_{3}^{+}$signal is almost suppressed [see Fig. 1(b)].

\section{Response of the LiF detector as a function of the $\mathrm{X}$-ray dose}

To define the PL response of the LiF detector, we used the diffraction pattern obtained in the first experiment [see Fig. 2(a)]. Fig. 3(a) shows a PL image of the diffraction pattern recorded on the $\mathrm{LiF}$ detector. Because of the high spatial resolution and the large field of view of the LiF detector, the diffraction pattern with a large number of well resolved fringes 
LiF image (from LSM700)

(a)



Horizontal profile

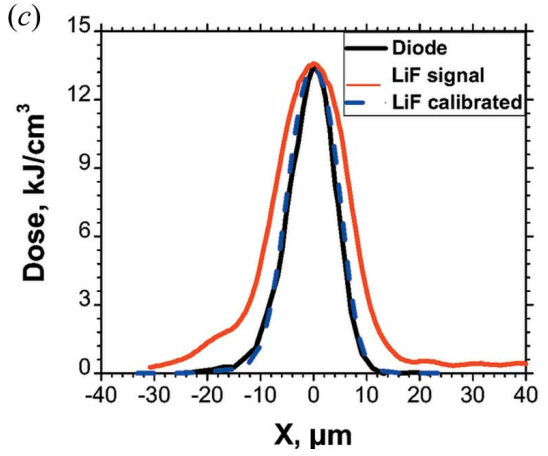

Vertical profile

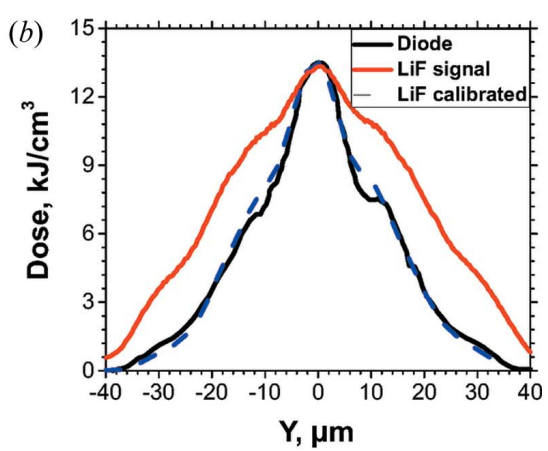

(d)

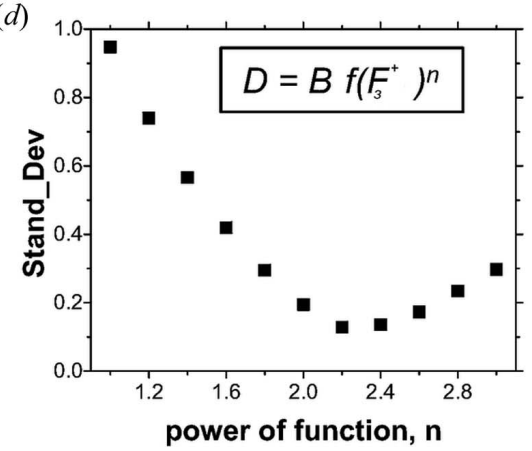

Figure 3

(a) A confocal laser-scanning microscope image of the irradiated LiF crystal behind the $100 \mu \mathrm{m}$ slit. $(b, c)$ Vertical and horizontal profiles of the X-ray dose distribution. The solid black line represents the diode measurements, the solid red line represents the LiF data normalized to the diode one, and the dashed blue line represents the $\mathrm{LiF}$ data calibrated by using the crystal response function found in Section 4. Black dashed lines in $(a)$ illustrate the areas over which the profiles were measured. (d) Dependence of the standard-deviation value between the diode and $\mathrm{LiF}$ data on the power index $n$ of the response function.

is clearly seen. The fringes in the horizontal direction are numerous and stem from a small slit size while the fringes in the vertical direction are few as a result of a widely opened vertical slit.

The spatial distribution of the beam obtained using a diode and a $\mathrm{LiF}$ crystal (along the same part of the diffraction pattern as had been scanned by the photodiode) is presented in Figs. 3(b) and 3(c) (black and red lines) in the horizontal and vertical directions, respectively. It can be seen that the obtained data differ considerably for the detectors. Since the LSM signal depends on the concentration of the $\mathrm{F}_{2}$ and $\mathrm{F}_{3}^{+}$ $\mathrm{CCs}$, we can conclude that the response of the $\mathrm{LiF}$ detector on the accumulated dose is nonlinear.

These days, the physics of CCs generation is not completely clear in relation to the dose/fluence of the ionizing radiation but it is known that the mechanism of formation of $F$ centers is statistical. In order to obtain metastable states which emit in the visible range (such as $\mathrm{F}_{2}, \mathrm{~F}_{3}^{+}$), the $\mathrm{F}$ centers should be accumulated. The formation of single centers can be described by a semi-empirical function (Baldacchini et al., 2005),

$$
f(D, P)=k(D)^{1 / 2}\left[1+\log \left(1+\frac{P}{P_{\mathrm{s}}}\right)^{2}\right]
$$

where $f(D, P)$ is the CC concentration $\left(1 \mathrm{~cm}^{-3}\right), k$ is the coefficient indicating the F-type CCs density, $D$ is the X-ray dose $\left(\mathrm{J} \mathrm{cm}^{-3}\right), P$ is the dose rate and $P_{\mathrm{s}}$ is the X-ray flux power threshold.

Equation (1) shows that the response function of the crystal is not linear with respect to the absorbed dose. Statistical accumulation of single centers is needed for the accumulation of more complex defects in the crystal lattice $\left(\mathrm{F}_{2}\right.$ and $\left.\mathrm{F}_{3}^{+}\right)$. If we assume that these events are independent, then the response function of the LiF crystal can be approximated as a power law. This allows us to obtain a quantitative dose distribution based on the number of observable $\mathrm{F}_{3}^{+}$ CCs as

$$
D=B f\left(\mathrm{~F}_{3}^{+}\right)^{n}
$$

where $D$ is the deposed dose, $B$ represents a coefficient (representing together the probability of CC creation by $500 \mathrm{eV}$ photons and the emissivity of the $\mathrm{F}_{3}^{+} \mathrm{CCs}$ fluorescence), $n$ is the power index and $f\left(\mathrm{~F}_{3}^{+}\right)$is the $\mathrm{F}_{3}^{+}$centers concentration $\left(\mathrm{cm}^{-3}\right)$,

$$
f\left(\mathrm{~F}_{3}^{+}\right)=\frac{I_{\mathrm{LSM} 700}}{F_{\text {scan }}},
$$

where $I_{\text {LSM700 }}$ is the gray value (counts of the microscope) and $F_{\text {scan }}$ is the microscope function.

In the work of Bonfigli et al. (2010), LiF crystals were irradiated by soft $\mathrm{X}$-rays emitted by a laser-plasma source in the energy range from 0.05 to $2 \mathrm{keV}$ (maximum intensity for $1 \mathrm{keV}$ ). The same dependence [equation (2)] was used for the fitting of the experimental points. It was found that $n=2.2$ for X-ray fluxes up to $120 \mu \mathrm{J} \mathrm{cm}^{-2}$, which corresponds to the accumulated dose of $1.2 \mathrm{~kJ} \mathrm{~cm}^{-3}$ in $\mathrm{LiF}$ [see equation (2)] for $\mathrm{F}_{3}^{+}$-type CCs.

Applying the response function [in the form of equation (2)] with a variable exponent $n$ to the beam profile data obtained using the LiF crystal [Figs. 3(b) and 3(c), red lines], we found the best fitting exponent value of $n=2.2$ at which the diode and the crystal data are in good agreement. Fig. 3(d) shows the dependence of the standard deviation on the exponent $n$ between the diode and LiF data in our experiment. As one can see, the best correlation between the diode and LiF data is observed at $n=2.2$ [see Figs. $3(b)$ and $3(c)$, blue and black lines] that corresponds to the minimum deviation in Fig. 3(d). Finally, equations (2) and (3) can be rewritten in the form

$$
D=B\left(\frac{I_{\mathrm{LSM} 700}}{F_{\text {scan }}}\right)^{2.2}
$$


Thus, we found that, for the monochromatic radiation with an energy of $500 \mathrm{eV}$, the response function of the $\mathrm{LiF}$ detector coincides with that obtained for polychromatic laser-plasma radiation [in the work of Bonfigli et al. (2010)] within a similar range of absorbed dose.

\section{Evaluation of LiF detector dynamic range}

Conventional X-ray detectors such as CCDs, IPs, radiochromic films and diodes typically provide a dynamic range of not more than $\sim 10^{5}$ in a single exposure. Here we specify the dynamic range of a detector as the ratio of the saturation (or damage) threshold to the minimum signal being recognized over the noise level of the same detector. In the following, we evaluate the dynamic range of the $\mathrm{LiF}$ detector. To do this, a pinhole was put at a distance of $12 \mathrm{~mm}$ upstream of the crystal for the observation of diffraction patterns in the far zone [see Fig. 2(b)]. The exposure time of the crystal was $100 \mathrm{~min}$ for the accumulation of the maximum dose on the detector.

It is evident that if we try to determine the dynamic range in one frame it is limited by the dynamic range of the readout system (this value is $6 \times 10^{4}$ for LSM700). However, we were able to vary the parameters of the microscope such as the gain of the PMT and the laser power to increase or decrease the signal value, and then combine both readouts to build a joint wide-range image. The corresponding apparatus functions linking the PL signal measured at different PMT gain and laser power to an actual accumulated dose were obtained for the LSM700 microscope in the frame of this work and are given in Appendix $A$. Thus, we can extend the dynamic range of the entire recording system (readout system $+\mathrm{LiF}$ crystal) to the dynamic range of the $\mathrm{LiF}$ detector. The technique becomes possible because the $\mathrm{LiF}$ detector can be read out many times. At first look, it may be expected that the excitation of CCs during the first readout decreases the total signal for future readings, but it is found that the signal decreases less than $0.1 \%$ after every scan. This allows a low signal to be observed as well as an extremely high one by varying the scanning parameters. On the contrary, such an approach is impossible for CCDs and IPs because the signal is wiped out after the first scan.

Using this technique, the full intensity distribution of the $\mathrm{X}$-ray beam passing the $5 \mu \mathrm{m}$ pinhole was observed and is given in Fig. 4. If we consider the frame $(a)$ obtained at gain $=$

Gain 360

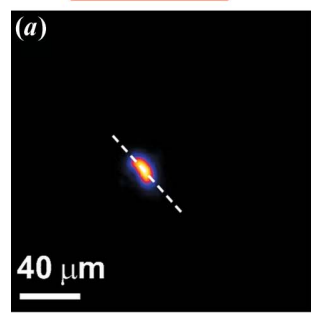

\section{Gain 740}

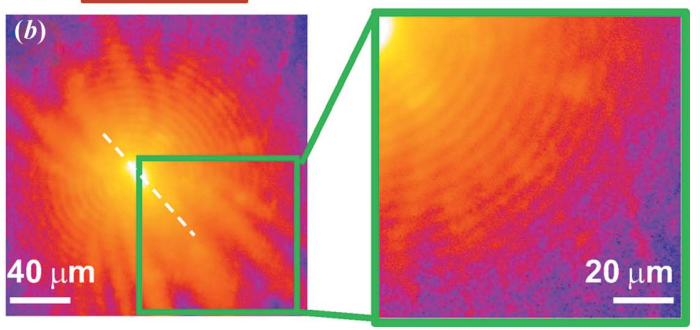

Figure 4

Diffraction images retrieved from the same exposure of LiF detector by LSM700 microscope with gain $=360(a)$ and gain $=740(b)$.

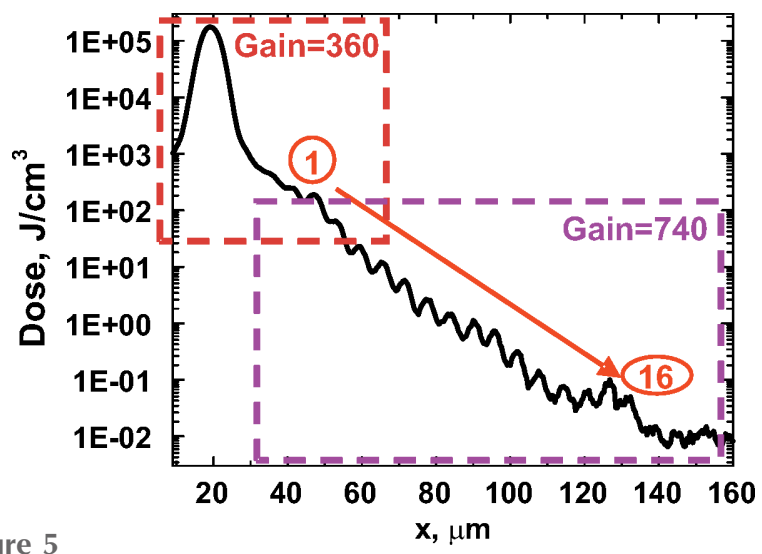

The dose distribution restored along the dashed white line in Fig. 4 (after applying the crystal-response function found in Section 4).

360 , one can only observe the central part of the beam, while the diffraction rings are not visible. Then, with the increase of the gain to 740, the central part of the image becomes saturated while the number of visible fringes increases [see Fig. 4(b), green frame].

It should be emphasized that we did not see the expected form (circle) of the signal behind the pinhole on the detector. This fact can be associated with instability of the beam in time (for long exposures).

Fig. 5 shows the dose profile of the X-ray radiation restored along the dashed white line in Fig. 4, which was found by using the previously obtained equation (4) for the response function of LiF. It is seen that 16 diffraction fringes are clearly distinguishable. The last ring has a dose of $2 \times 10^{-2} \mathrm{~J} \mathrm{~cm}^{-3}$, while the maximum dose is $1.7 \times 10^{5} \mathrm{~J} \mathrm{~cm}^{-3}$. Thus, we can conclude that the LiF detector allows one to build up a single exposure $\mathrm{X}$-ray image with a dynamic range of not less than $10^{7}$. The absorbed dose in the last visible diffraction ring corresponds to the sensitivity threshold of the LiF crystal. This value is four to five orders more than for a CCD; however, the advantage of $\mathrm{LiF}$ is the possibility to use it in high-intensity fields.

It should also be emphasized that the $\mathrm{LiF}$ dynamic range does not depend on the photon energy or accumulated dose, but might be limited in the case of high beam fluxes. While the $\mathrm{LiF}$ detector is still operational at enormous accumulated doses over several $\mathrm{MJ} \mathrm{cm}{ }^{-3}$, the high dose rate delivered by XFEL pulses may already cause the ablation of the detector surface at a few $\mathrm{kJ} \mathrm{cm}^{-3}$ per femtosecond pulse. In our experiments, the dose rate did not exceed $30 \mathrm{~J} \mathrm{~cm}^{-3}$ per synchrotron pulse. It is worth noting that the maximum dose for the damage to $\mathrm{LiF}$ crystal was not accumulated in the experiment. Thus, the potential dynamic range of the crystal is more significant than what we have registered.

\section{Spatial resolution and imaging capability of the crystal}

In experiments where the structure of an object should be determined at the nanometre 
scale, the detector must also have a high spatial resolution. Potentially, a $\mathrm{LiF}$ crystal can have a spatial resolution comparable with atomic size. However, practically this value is limited by both the spatial resolution of the readout system and the size of the secondary electron cloud. The latter effect strongly depends on the X-ray energy. The physics of this process are discussed in the work by Grum-Grzhimailo et al. (2017) in detail. As an example, the spatial resolution of the $\mathrm{LiF}$ crystal achieved for hard X-ray photons of an XFEL (10.1 keV) was $\sim 1.2 \mu \mathrm{m}$ (Pikuz et al., 2015), while for a soft X-ray laserplasma source the diffraction pattern was obtained with a resolution of 0.05 $0.075 \mu \mathrm{m}$ (Oliva et al., 2008; Ustione et al., 2006) by using scanning near-field optical microscopy.

For our conditions, the radius of the secondary electron cloud is expected to be less than $10 \mathrm{~nm}$ for the X-ray beam with $500 \mathrm{eV}$ energy and cannot affect the resolution power. Therefore, in our experiment, the spatial resolution of the $\mathrm{LiF}$ detector is limited by the readout system. We scanned the irradiated LiF crystal using the immersion objective with a numerical aperture value of 1.4 and an excitation wavelength of $488 \mathrm{~nm}$ that allowed us to achieve a spatial resolution of $\sim 0.2 \mu \mathrm{m}$.

The X-ray beam profiles were obtained in the planes at different distances from the pinhole (see Fig. 6, left column). The spatial distribution of the beam spot with a size of $4 \mu \mathrm{m}$ was recorded. Fig. 6 shows features in the central region of the beam (for $z=1.3 \mathrm{~mm}$ and $z=1.5 \mathrm{~mm}$ ). Because of the provided spatial resolution of the $\mathrm{LiF}$ detector, we could observe inhomogeneities of the spatial distribution of the $\mathrm{X}$-ray beam in various planes. The LiF image profile shows the features in the $\mathrm{X}$-ray beam distribution $(\sim 0.8 \mu \mathrm{m}$ in size $)$ in Fig. 6(c). This value is greater than can be expected from both the blurring effect by the secondary electron cloud (several nanometres) and the spatial resolution of the LSM700 microscope.

We also simulated the propagation of the X-ray beam through the setup to compare it with the experimental beam distribution. The modeling was performed with a software framework for coherent and partially coherent X-ray wavefront propagation simulations - WavePropaGator (Samoylova et al., 2016), which is a development of the calculation methods implemented in $S R W$ and which is available through open-access code (Chubar et al., 2008). The results of the modeling are presented in Fig. 6 (images in red frames). It can be seen that the spatial distribution of the beam is similar for all $z$ planes. However, the amplitudes and symmetry of the experimental beam profiles differ from the modeling (see Fig. 6, black and red lines on the graphics). The size of the features in the simulated beam profile is the same as for the experimental data [see Fig. 6(c)]. Thus, we expect that for the water-window X-ray range the real spatial resolution of the LiF detector should be better than we were able to demonstrate in particular experimental configurations. Finally, it should be noted that in this article we provide just an example of LiF imaging capabilities and not careful measurements of the spatial resolution limit.

\section{Summary}

An experiment for the calibration of an X-ray lithium fluoride detector was performed at the P04 beamline at PETRA III. As a result, the metrological properties of the $\mathrm{LiF}$ crystal were retrieved. In particular, the $\mathrm{LiF}$ detector response function was determined for the X-ray deposed dose up to $\sim 14 \mathrm{~kJ} \mathrm{~cm}^{-3}$. This function is well described by a power-law dependence (power index $n=2.2$ ). Using this dependence, we demonstrated a dynamic range of $\mathrm{LiF}$ of up to $10^{7}$ for our experimental conditions. Also, the threshold for the CCs creation was estimated as $2 \times 10^{-2} \mathrm{~J} \mathrm{~cm}^{-3}$. This value corresponds to the dose in the last visible diffraction fringes for the experiment with pinhole diffraction. 
It was also demonstrated that the sub-micrometre imaging capability of the X-ray beam distribution is achieved. LiF images showed the features in the X-ray beam distribution $(\sim 0.8 \mu \mathrm{m}$ in size) that correspond to the theoretical calculations. These data make it possible to extend the use of $\mathrm{LiF}$ as a detector for the diagnostic and characterizing of X-ray beams in extreme-intensity experiments on the study of nanoscale objects.

\section{APPENDIX $A$}

In addition, we also present the microscope calibration functions because there is no such data for the Carl Zeiss LSM700 provided by the vendor. In particular, we found the dependence of microscope counts $I_{\text {LSM700 }}$ (PMT signal) on the PMT gain and pumping laser power. For this, we scanned the test plate with different microscope parameters.

Fig. 7(a) shows the dependence of the PMT signal $I_{\mathrm{LSM} 700}$ on the laser power (keeping PMT gain parameter $G=$ constant). It is well approximated by a linear dependence with a factor of 0.01 with respect to the reference point at laser power $=100 \%$, where the signal was normalized to 1 ,

$$
\begin{aligned}
I_{\mathrm{LSM} 700}\left(P_{\text {las }}, G\right. & =\text { const }) \\
& =A_{\text {las }} P_{\text {las }} I_{\text {LSM700 }}\left(P_{\text {las }}=100 \%, G=\mathrm{const}\right) \\
& =0.01 P_{\text {las }} I_{\text {LSM } 700}\left(P_{\text {las }}=100 \%, G=\text { const }\right),
\end{aligned}
$$

where $I_{\mathrm{LSM} 700}\left(P_{\text {las }}, G=\right.$ const $)$ is the PMT signal with the arbitrary value $P_{\text {las }}$ (counts per pixel in the microscope data), $A_{\text {las }}$ is an approximation coefficient of the laser, $P_{\text {las }}$ is the laser-power value and $I_{\mathrm{LSM} 700}\left(P_{\text {las }}=100 \%, G=\right.$ const $)$ is the PMT signal with $P_{\text {las }}=100 \%$.

Fig. 7(b) shows the dependence of the signal value on the PMT gain. It is well approximated by an exponential dependence with an exponent factor of $t=88$ with respect to the reference point at $G=360$, where the signal was normalized to 1 . So the PMT signal can be presented as

$$
\begin{aligned}
& I_{\mathrm{LSM} 700}\left(G, P_{\text {las }}=\text { const }\right) \\
& \quad=A_{G} \exp (G / t) I_{\mathrm{LSM} 700}\left(G=360, P_{\text {las }}=\text { const }\right) \\
& \quad=0.017 \exp (G / 88) I_{\mathrm{LSM} 700}\left(G=360, P_{\text {las }}=\text { const }\right),
\end{aligned}
$$

where $I_{\mathrm{LSM} 700}\left(G, P_{\text {las }}=\right.$ const $)$ is the PMT signal with arbitrary value gain $G$ (counts of the microscope), $A_{G}$ is an approx-
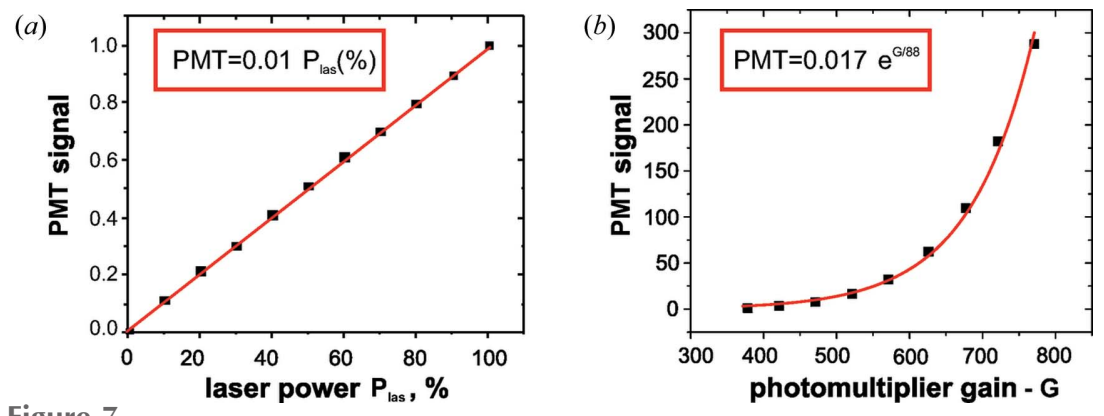

Instrumental calibration functions for the Carl Zeiss LSM700 fluorescent microscope. (a) PMT signal normalized to a signal with laser power $=100 \%$ versus illuminating laser power. (b) PMT signal normalized to a signal with gain $=360$ versus PMT gain. imation coefficent of the PMT gain, $t$ is an exponent factor and $I_{\text {LSM } 700}\left(G=360, P_{\text {las }}=\right.$ const $)$ is the $\mathrm{F}_{3}^{+}$centers concentration $\left(\mathrm{cm}^{-3}\right)$.

If we vary both the PMT gain and laser power parameters, the total PMT signal $I_{\mathrm{LSM} 700}$ is

$$
\begin{aligned}
I_{\mathrm{LSM} 700}\left(P_{\text {las }}, G\right)= & A_{\text {las }} P_{\text {las }} A_{G} \exp (G / t) \\
& \times I_{\mathrm{LSM} 700}\left(P_{\text {las }}=100 \%, G=360\right) .
\end{aligned}
$$

Or, using equations (5) and (6),

$$
\begin{aligned}
I_{\mathrm{LSM} 700}\left(P_{\text {las }}, G\right)= & F_{\text {scan }} I_{\mathrm{LSM} 700}\left(P_{\text {las }}=100 \%, G=360\right) \\
= & 0.01 P_{\text {las }} 0.017 \exp (G / 88) \\
& \times I_{\text {LSM700 }}\left(P_{\text {las }}=100 \%, G=360\right) .
\end{aligned}
$$

Thus, equation (4) in the main section of this article for the absorbed dose $D$ in LiF can be represented in the form

$$
D=B\left[\frac{I_{\mathrm{LSM} 700}\left(P_{\text {las }}, G\right)}{F_{\text {scan }}}\right]^{2.2}=B\left[\frac{I_{\mathrm{LSM} 700}\left(P_{\text {las }}, G\right)}{A_{\text {las }} P_{\text {las }} A_{G} \exp (G / t)}\right]^{2.2},
$$

which helps to establish a cross-correspondence in the data measured at different microscope settings.

Using the reference point at $P_{\text {las }}=100 \%$ and $G=360$ and by comparison with calibrated $\mathrm{X}$-ray photodiode data as shown in Fig. 3, the parameter $B$ representing together the probability of $\mathrm{CC}$ creation by $500 \mathrm{eV}$ photons and the emissivity of the $\mathrm{F}_{3}^{+} \mathrm{CCs}$ fluorescence has been found to be $3.7 \times 10^{-7}$. Finally, the absolute dose $D$ value distribution can be revealed for any LSM parameters providing cross-calibration for the data taken with different laser pumping power and PMT gain,

$$
D=3.7 \times 10^{-7}\left[\frac{I_{\mathrm{LSM} 700}\left(P_{\mathrm{las}}, G\right)}{0.01 P_{\mathrm{las}} \times 0.017 \exp (G / 88)}\right]^{2.2} .
$$

\section{Funding information}

The following funding is acknowledged: Ministry of Science and Higher Education of the Russian Federation (in the frame of Photon Science Experiment Program and the State assignment No. 01201357846 to JIHT RAS); Foundation for the Advancement of Theoretical Physics and Mathematics

'BASIS' (grant No. 18-2-6-67-1 to S. Makarov); Helmholtz Associations Initiative and Networking Fund (Grant No. HRSF-0002 to M. Rose, S. Lazarev, I. Zaluzhnyy and I. Vartanyants); German Federal Ministry of Education and Research (BMBF) (Grant No. 05K19PC2 to A. Rosenhahn); Competitiveness Enhancement Program of National Research Tomsk Polytechnic University.

\section{References}

Almaviva, S., Baldacchini, G., Piccinini, M. \& Montereali, R. M. (2007). J. Non-Cryst. Solids, 353, 687-691. 
Baldacchini, G., Bollanti, S., Bonfigli, F., Flora, F., Di Lazzaro, P., Lai, A., Marolo, T., Montereali, R. M., Murra, D., Faenov, A., Pikuz, T., Nichelatti, E., Tomassetti, G., Reale, A., Reale, L., Ritucci, A., Limongi, T., Palladino, L., Francucci, M., Martellucci, S. \& Petrocelli, G. (2005). Rev. Sci. Instrum. 76, 113104.

Baldacchini, G., Bonfigli, F., Faenov, A., Flora, F., Montereali, R. M., Pace, A., Pikuz, T. \& Reale, L. (2003). J. Nanosci. Nanotech. 3, 483-486.

Bonfigli, F., Gaudio, P., Lupelli, I., Nichelatti, E., Richetta, M., Vincenti, M. A. \& Montereali, R. M. (2010). IOP Conf. Ser. Mater. Sci. Eng. 15, 012025.

Bonfigli, F., Hampai, D., Dabagov, S. B. \& Montereali, R. M. (2016). Opt. Mater. 58, 398-405.

Bonfigli, F., Hartley, N., Inubushi, Yu., Koenig, M., Matsuoka, T., Makarov, S., Montereali, R. M., Nichelatti, E., Ozaki, N., Piccinini, M., Pikuz, S., Pikuz, T., Sagae, D., Vincenti, M. A., Yabashi, M. \& Yabuuchi, T. (2019). Proc. SPIE, 11035, 110350N.

Chubar, O., Couprie, M. E., Labat, M., Lambert, G., Polack, F. \& Tcherbakoff, O. (2008). Nucl. Instrum. Methods Phys. Res. A, 593, 30-34.

Faenov, A., Matsubayashi, M., Pikuz, T., Fukuda, Y., Kando, M., Yasuda, R., Iikura, H., Nojima, T., Sakai, T., Shiozawa, M., Kodama, R. \& Kato, Y. (2015). High Power Laser Sci. Eng. 3, e27.

Faenov, A., Pikuz, T., Fucuda, Yu., Kando, M., Kotaki, H., Homma, T., Kawase, K., Scobelev, I., Gasilov, S., Kawachi, T., Daido, H., Tajima, T., Kato, Y. \& Bulanov, S. (2010). Jpn J. Appl. Phys. 49, 06GK03.

Faenov, A. Ya., Kato, Y., Tanaka, M., Pikuz, T. A., Kishimoto, M., Ishino, M., Nishikino, M., Fukuda, Y., Bulanov, S. V. \& Kawachi, T. (2009). Opt. Lett. 34, 941-943.

Faenov, A. Y., Pikuz, T. A., Mabey, P., Albertazzi, B., Michel, T., Rigon, G., Pikuz, S. A., Buzmakov, A., Makarov, S., Ozaki, N., Matsuoka, T., Katagiri, K., Miyanishi, K., Takahashi, K., Tanaka, K. A., Inubushi, Y., Togashi, T., Yabuuchi, T., Yabashi, M., Casner, A., Kodama, R. \& Koenig, M. (2018). Sci. Rep. 8, 16407.

Grum-Grzhimailo, A. N., Pikuz, T., Faenov, A., Matsuoka, T., Ozaki, N., Albertazzi, B., Pikuz, S., Inubushi, Yu., Yabashi, M., Tono, K., Yumoto, H., Ohashi, H., Ishikawa, T. \& Kodama, R. (2017). Eur. Phys. J. D, 71, 69.

Kurobori, T. \& Matoba, A. (2014). Jpn J. Appl. Phys. 53, $02 B D 14$.

Kurobori, T., Miyamoto, Y., Maruyama, Y., Yamamoto, T. \& Sasaki, T. (2014). Nucl. Instrum. Methods Phys. Res. B, 326, 76-80.

Montereali, R. M., Ampollini, A., Picardi, L., Ronsivalle, C., Bonfigli, F., Libera, S., Nichelatti, E., Piccinini, M. \& Vincenti, M. A. (2018). J. Lumin. 200, 30-34.
Oliva, C., Ustione, A., Almaviva, S., Baldacchini, G., Bonfigli, F., Flora, F., Lai, A., Montereali, R. M., Faenov, A., Pikuz, T. A., Francucci, M., Gaudio, P., Martellucci, S., Richetta, M., Reale, L. \& Cricenti, A. (2008). J. Microsc. 229, 490-495.

Piccinini, M., Nichelatti, E., Ampollini, A., Picardi, L., Ronsivalle, C., Bonfigli, F., Libera, S., Vincenti, M. A. \& Montereali, R. M. (2017). Europhys. Lett. 117, 37004.

Pikuz, T. A., Faenov, A., Ozaki, N., Matsuoka, T., Albertazzi, B., Hartley, N. J., Miyanishi, K., Katagiri, K., Matsuyama, S., Yamauchi, K., Habara, H., Inubushi, Y., Togashi, T., Yumoto, H., Ohashi, H., Tange, Y., Yabuuchi, T., Yabashi, M., Grum-Grzhimailo, A., Casner, A., Skobelev, I., Makarov, S., Pikuz, S., Rigon, G., Koenig, M., Tanaka, K. A., Ishikawa, T. \& Kodama, R. (2018). Matter Radiat. Extremes, 3, 197-206.

Pikuz, T., Faenov, A., Matsuoka, T., Matsuyama, S., Yamauchi, K., Ozaki, N., Albertazzi, B., Inubushi, Y., Yabashi, M., Tono, K., Sato, Y., Yumoto, H., Ohashi, H., Pikuz, S., Grum-Grzhimailo, A. N., Nishikino, M., Kawachi, T., Ishikawa, T. \& Kodama, R. (2015). Sci. Rep. 5, 17713.

Rose, M., Senkbeil, T., von Gundlach, A. R., Stuhr, S., Rumancev, Ch., Dzhigaev, D., Besedin, I., Skopintsev, P., Loetgering, L., Viefhaus, J., Rosenhahn, A. \& Vartanyants, I. A. (2018). Opt. Express, 26, 1237-1254.

Rose, M., Skopintsev, P., Dzhigaev, D., Gorobtsov, O., Senkbeil, T., von Gundlach, A., Gorniak, T., Shabalin, A., Viefhaus, J., Rosenhahn, A. \& Vartanyants, I. (2015). J. Synchrotron Rad. 22, 819-827.

Ruiz-Lopez, M., Faenov, A., Pikuz, T., Ozaki, N., Mitrofanov, A., Albertazzi, B., Hartley, N., Matsuoka, T., Ochante, R., Tange, Y., Yabuuchi, T., Habara, T., Tanaka, K. A., Inubushi, Y., Yabashi, M., Nishikino, M., Kawachi, T., Pikuz, S., Ishikawa, T., Kodama, R. \& Bleiner, D. (2017). J. Synchrotron Rad. 24, 196204.

Samoylova, L., Buzmakov, A., Chubar, O. \& Sinn, H. (2016). J. Appl. Cryst. 49, 1347-1355.

Ustione, A., Cricenti, A., Bonfigli, F., Flora, F., Lai, A., Marolo, T., Maria Montereali, R., Baldacchini, G., Faenov, A., Pikuz, T. \& Reale, L. (2006). Appl. Phys. Lett. 88, 141107.

Viefhaus, J., Scholz, F., Deinert, S., Glaser, L., Ilchen, M., Seltmann, J., Walter, P. \& Siewert, F. (2013). Nucl. Instrum. Methods Phys. Res. A, 710, 151-154.

Voitovich, P., Kalinov, V. S., Runets, L. P., Stupak, A. P., Martynovich, F., Montereali, R. M. \& Baldacchini, G. (2013). J. Lumin. 143, 207-214. 